\title{
Estratégias de orientações psicoeducacionais para familiares de deficientes*
}

\section{Strategies of psyco-educational orientations for family members of handcaps}

\author{
Lúcia Pereira Leite ${ }^{1}$ \\ Sandra Eli Sartoreto de Oliveira Martins² \\ Simone Ghedini Costa Milanez ${ }^{3}$
}

\begin{abstract}
RESUMO
O presente trabalho teve como objetivo propiciar o estabelecimento de uma proposta de intervenção psicoeducacional, em encontros sistemáticos, com os respectivos pais e/ou familiares de deficientes, na tentativa de se estabelecer um "espaço" de discussão sobre as problemáticas relacionadas ao processo educacional. Participaram desse projeto 30 familiares de alunos com deficiências, sendo 21 pais de alunos com deficiência auditiva e 9 pais de alunos com deficiência física, que freqüentavam atendimento pedagógico no Centro de Estudos da Educação e Saúde (Cees Unesp/ Marília). Após a implementação do programa de orientações psicoeducacionais a familiares e, conseqüentemente, do acompanhamento das atividades realizadas, verificamos que a realização desse programa, além de orientar os familiares sobre os aspectos educacionais, favoreceu o esclarecimento de algumas questões concernentes ao desenvolvimento global de indivíduos com necessidades educacionais especiais; a população investigada confirmou a carência de programas de orientações psicoeducacionais em serviços públicos, manifestando dificuldade em ter acesso a profissionais e locais que oferecem esse tipo de suporte. Os re-
\end{abstract}

* O projeto contou com o apoio da Fundunesp-Proex, na compra de materiais de consumo, além de ter sido contemplado com um bolsista.

${ }^{1}$ Doutora em Educação/Departamento de Psicologia- FC- Unesp-Bauru.

${ }^{2}$ Doutoranda em Educação/Departamento de Educação Especial - FCC- Unesp- Marília.

${ }^{3}$ Doutora em Genética Humana/Departamento de Educação Especial -FCC-Unesp-Marília. 
sultados obtidos neste trabalho vêm reafirmar a importância de se implementar propostas de orientações com familiares, aliadas aos atendimentos com os indivíduos que apresentam necessidades educacionais especiais, como na literatura pesquisada. De acordo como proposto na abordagem sistêmica, entende-se que a intervenção psicoeducacional só será efetivada com sucesso quando deixar de focar apenas o indivíduo e se estender aos seus pares, principalmente àqueles que convivem diretamente com ele.

Palavras-chave: orientações psicopedagógicas, avaliação educacional, necessidades educacionais especiais.

\begin{abstract}
The current work had the aim to provide the establishment of a proposal of psyco-educational intervention, in systematic meetings, with the respective parents and/or family members of handcaps, attempting to establish a discussion "space" about the problems related to the educational process. Thirty family members of students with auditive disabilities took part in this project and nine parents of students with physical disabilities who attended the pedagogical service at the Center of Education and Health Studies (Cees - Unesp/ Marília). After the implementation of the program of psyco-educational orientation for family members and consequently, the follow up of the performed activities, we noticed that the achievement of this program besides orienting the family members about educational aspects, it favored the elucidation of some questions concerning the global development of individuals with special educational necessities; the studied population confirmed the lack of psyco-educational orientation programs in public service, showing difficulty in having access to professionals and locals who offer this kind of support. The obtained results in this work reaffirm the importance of implementing proposals of orientations with family members, together with the services with individuals who present special educational necessities, as in the researched literature. According to what was proposed in the systemic approach, it is understood that the psyco-educational intervention will only be effective successfully when it stops forcing the individual and extends itself to its pairs, mainly those who live directly with him.

Key-words: psyco-educational orientations, psyco-educational intervention, parents of handcaps.
\end{abstract}




\section{Introdução}

De acordo com BASSEDAS (1996), a família é tida como um sistema que possui uma função psicossocial de proteger os seus membros e uma função social de transmitir e favorecer a adaptação à cultura existente. Assim, cada família apresenta dada estrutura que se organiza a partir das demandas, interações e comunicações, as quais ocorrem tanto no seu interior, quanto no mundo exterior. Por sua vez, dentro da família existem vários subsistemas, como, por exemplo, o casal, pais e filhos, os filhos, entre outros. Esses subsistemas se relacionam entre si e com outros sistemas sociais - escola, igreja, clubes, associações etc. Essa estruturação social é mantida a partir dos limites, uns mais rígidos, outros mais flexíveis, impostos pelos sistemas e subsistemas, que protegem a manutenção de sua estrutura.

Segundo a autora, a criança formará naturalmente suas expectativas em torno de modelos e informações que a família lhe proporciona, iniciando, assim, sua escolaridade. No caso específico de crianças com necessidades educacionais especiais, sabe-se que a família nem sempre consegue lidar de maneira equilibrada com os problemas decorrentes das limitações ocasionadas pelas deficiências de seus filhos, inclusive os escolares. Desse modo, a falta de orientação e o despreparo dos familiares agravam a insegurança da criança no enfrentamento de suas atividades diárias, quer no âmbito escolar, quer no social e/ou familiar.

Ao se pensar na família enquanto um sistema composto de indivíduos interdependentes, entende-se que as ações a serem promovidas a cada um dos seus membros acabam por afetar seu pares, uma vez que a troca de experiências interacionais acarretará o desenvolvimento do vínculo parental.

Segundo CORREA e SERRANO (1999), é importante que os profissionais envolvidos com a educação de alunos com necessidades educacionais especiais (NEE's) entendam o conceito sistêmico de família, para estabelecer as estratégias de intervenção educacional. Essa abordagem supõe, entre outros aspectos, que não se deve centrar o trabalho exclusivamente com a população com NEE's, mas estendê-lo ao contexto familiar e ambiental.

É muito interessante quando os autores relatam, também, que antes de propor intervenções com os familiares, os profissionais devem compreender o ciclo de vida da família que possui uma criança com NEE, compreendendo seus medos, expectativas, procura de atendimentos, preocupações de aceitação social, cuidados especiais, entre outros.

Para assegurar o desenvolvimento da criança com NEE, os pais, na maioria das vezes, têm que se adaptar a novas condições sociais, o que irá 
provocar mudanças na estrutura familiar. Dependendo da necessidade da criança, essas modificações podem afetar, por exemplo: o ritmo de trabalho dos pais - que precisam dispor de um tempo maior para cuidarem do seu filho; a situação econômica - necessidade de disponibilidade para auxílio médico e educacional especializado; auxílio externo - para transporte, locomoção, ajuda com cuidados pessoais e domésticos; o relacionamento do casal e dos outros filhos - havendo a necessidade de suporte psicológico, para enfrentar a rotina do cotidiano e superar o sentimento de culpa ou desânimo, entre outros fatores.

Para SOMMERSTEIN e WESSELS (1999), o sentimento negativista e pessimista sempre está presente na família de uma criança com NEE, pois esta recebe, muitas vezes, respostas do meio social que não apresentam condições favoráveis para o desenvolvimento global dessa criança. Muitas vezes, os próprios profissionais, no processo de avaliação, enfatizam aos pais mais as dificuldades do que as potencialidades percebidas na criança, criando uma baixa expectativa de sucesso para a realização de atividades educacionais.

Uma das maneiras apontadas pelos autores para lidar com essa prática constitui-se em favorecer, aos pais, condições para que compreendam que, apesar da diferença no ritmo de desenvolvimento apresentada por uma criança com NEE, ela poderá, com ajuda de profissionais e dos familiares, participar de estratégias educacionais que promovam seu desenvolvimento. De acordo com VIGOTSKY (2001), isso pode ser viabilizado a partir do resgate de suas potencialidades, ou seja, do que consegue fazer sozinha ou com parceiros mais capazes, para realizar a tarefa proposta.

É importante, então, que se estabeleça um elo de comunicação em que os profissionais envolvidos priorizem a orientação aos pais sobre os procedimentos favoráveis ao desenvolvimento global de seus filhos, a partir das necessidades apresentadas por eles.

FONSECA (1995) salienta que os serviços oferecidos aos familiares de deficientes devem possibilitar que os pais sejam capacitados para favorecer as aprendizagens básicas para seus filhos, desde os primeiros anos de vida. Segundo o autor, "os pais terão de ser preparados para os problemas de higiene corporal, de disciplina, de isolamento, de insegurança, de recreação, de espaço habitacional, de estimulação, de mobilidade, de aprendizagem, etc. Neste aspecto, o apoio aos pais deve transcender a mera observação médica” (p. 213).

Salienta, ainda, que os pais muitas vezes recebem orientações de difícil compreensão, ou pela adoção de termos técnicos ou pelo uso inadequado de expressões pouco objetivas, o que dificulta o entendimento e o manejo das práticas de intervenção com filho deficiente. 
$\mathrm{O}$ emprego de um linguajar adequado e o atendimento às expectativas das necessidades reais apresentadas pelos familiares são premissas que devem nortear qualquer proposta de intervenção educacional. Muito freqüentemente, incorre-se no erro de se estabelecer programas de orientações aos pais, planejados somente por profissionais sem consulta prévia aos familiares, desestimulando a sua participação. Com isso, deixa-se de facilitar o estabelecimento de um espaço de interlocução, em que os pais possam encontrar respostas às suas dúvidas, com objetividade e respaldo teórico.

Refletindo sobre as considerações apresentadas, além do convívio profissional com familiares de alunos com necessidades educacionais especiais, pareceu-nos importante viabilizar orientações aos familiares de deficientes, uma vez que essa população nos procurava querendo encaminhamentos, respostas, ajuda sobre questões encontradas no trato diário com seus filhos, principalmente no âmbito educacional.

Assim, elaboramos um projeto de extensão universitária, que teve como objetivo favorecer a implementação de um programa de orientação aos familiares de crianças e/ou adolescentes com necessidades educacionais especiais, a partir de temas escolhidos pelos pais e nos apontamentos da literatura pertinente, que estivessem relacionados à área psicopedagógica.

Esse programa, então, consistiu em propiciar o estabelecimento de uma proposta de intervenção psicoeducacional, em encontros sistemáticos durante um ano letivo, com esses pais e/ou familiares, na tentativa de se estabelecer um "espaço" de discussão sobre as problemáticas, relacionadas ao desenvolvimento psicossocial de seus filhos. Isso nos pareceu importante, pois a maioria desses pais apresentava situação econômica desfavorável e baixo grau de escolaridade, não se beneficiando de nenhuma proposta de orientação psicoeducacional, por terem dificuldades de encontrar tal tipo de auxílio em serviços comunitários.

\section{Metodologia}

\section{Sujeitos}

Participaram desse projeto 30 familiares de alunos com deficiências, sendo 21 pais de alunos com deficiência auditiva e 9 pais de alunos com deficiência física, que freqüentavam atendimento pedagógico no Centro de Estudos da Educação e Saúde (Cees - Unesp/ Marília). 


\section{Local}

O trabalho de extensão e pesquisa desenvolveu-se no Centro de Estudos de Educação e Saúde, vinculado à Faculdade de Filosofia e Ciências (FFC) da Unesp (Campus de Marília), que tem como principal objetivo fornecer apoio às atividades de ensino, pesquisa e extensão. Os atendimentos prestados voltam-se para temáticas relativas ao desenvolvimento e educação de indivíduos com necessidades especiais, decorrentes de alterações genéticas, morfofisiológicas e/ou psicossociais.

O Cees conta com o apoio de uma equipe de profissionais multidisciplinares, os quais auxiliam no desenvolvimento dos trabalhos de extensão e pesquisa da universidade, bem como no oferecimento de serviços à comunidade de Marília e região, nas áreas de fonoaudiologia, pedagogia, psicologia, assistência social, fisioterapia, terapia ocupacional, entre outras.

O programa de orientação psicoeducacional foi desenvolvido, durante um $a^{4}{ }^{4}$, por três professoras do Departamento de Educação Especial, da Faculdade de Filosofia e Ciências, campus de Marília, sendo duas dessas responsáveis pela disciplina Prática de Ensino e Estágio Supervisionado, nas áreas de deficiência auditiva e física. Também teve a colaboração de uma aluna do curso de Pedagogia, para auxiliar no desenvolvimento do programa.

\section{Desenvolvimento do programa de orientações psicoeducacionais}

O trabalho de intervenção psicopedagógica foi desenvolvido em três etapas. Na primeira delas, foi elaborado um questionário semi-estruturado com a finalidade de levantar temas de acordo com os interesses dos familiares, no manejo das práticas educacionais a serem desenvolvidas com os seus filhos.

O questionário foi composto de três questões semi-abertas e uma de múltipla escolha, e aplicado aos participantes desse estudo. As questões procuravam conhecer as expectativas dos familiares, referentes ao desenvolvimento psicoeducacional do deficiente.

A aplicação do questionário ocorreu de forma individual, por meio de entrevistas com os familiares de deficientes auditivos e deficientes físicos, no Cees. Durante as entrevistas, foram lidas as questões, anotando-se as respostas dos sujeitos para cada uma delas. Tal procedimento foi utilizado com intuito de evitar possíveis desencontros de informações quanto ao entendimen-

\footnotetext{
${ }^{4}$ O período de execução do projeto compreendeu de agosto de 2002 a setembro de 2003.
} 
to do que estava sendo proposto, pois alguns dos familiares não eram alfabetizados, o que impossibilitava a coleta por escrito. No decorrer das entrevistas, buscou-se, também, esclarecer aos pais a proposta de intervenção psicopedagógica, como obter sua autorização para a participação no projeto e posterior divulgação dos seus dados ${ }^{5}$.

A segunda etapa constou da tabulação e análise das informações coletadas nos referidos questionários. As temáticas levantadas nas entrevistas foram: sexualidade, inclusão educacional, desenvolvimento geral, aspectos relacionados ao atendimento desenvolvido no Cees, problemas de relacionamento social, aspectos referentes à deficiência e comportamento do deficiente na família.

A partir desse levantamento, realizou-se um agendamento com profissionais de diferentes áreas de atuação, envolvidos com as especificidades apontadas, para proferirem palestras aos familiares. Na consulta feita aos referidos profissionais, destacou-se a importância de estabelecerem momentos de reflexão sobre o conteúdo discutido com os pais, criando oportunidades para que eles pudessem expor suas dúvidas e opiniões a respeito.

A terceira etapa consistiu no oferecimento de encontros com profissionais especializados em cada área levantada, como ilustrado no quadro da página seguinte.

Durante a realização das palestras, foram propiciadas situações para que os familiares se manifestassem, expondo suas dúvidas sobre os temas trabalhados. Ressaltamos, informalmente, que, durante os encontros, a maioria dos familiares apresentava poucos conhecimentos sobre os conteúdos abordados. Isso foi identificado por meio da interação dos pais com os profissionais, a partir da manifestação de relatos sobre o cotidiano dos filhos, em diversas situações educacionais e ambientes sociais.

Dessa forma, os relatos indicaram que as palestras, segundo as opiniões da maioria dos participantes, atingiram os objetivos propostos, estabelecendo um "espaço" que favoreceu a manifestação dos familiares e a obtenção de informações a respeito de suas dúvidas mais freqüentes.

Em virtude de os familiares demonstrarem interesse em assuntos relacionados ao desenvolvimento educacional de seus filhos, foi realizado um levantamento da realidade educacional atual, bem como da vida pregressa escolar dessa população, a partir da investigação dos dados escolares apresentados nos prontuários do Cees. Todavia, nessa análise documental, percebemos que os dados, na maioria das vezes, estavam incompletos e desatualizados.

${ }^{5}$ Conforme indicado pela Resolução 196/96, do Conselho Nacional de Saúde, que propõe a normalização para pesquisas realizadas com seres humanos. 
LEITE, L. P. et al. Estratégias de orientações psicoeducacionais...

QUADRO 1: TEMÁTICAS ABORDADAS NAS SESSÕES DE ORIENTAÇÕES PSICOEDUCACIONAIS

\begin{tabular}{|c|c|c|}
\hline Área trabalhada & Conteúdo & Profissional \\
\hline - Inclusão Educacional & $\begin{array}{l}\text { - Conceituação das necessidades educacionais especiais } \\
\text { (NEE) } \\
\text { - Política educacional de atendimento ao aluno com NEE } \\
\text { - Modalidades educacionais para o aluno NEE } \\
\text { - Análise situacional }\end{array}$ & Pedagoga \\
\hline $\begin{array}{l}\text { - Aprendizagem e } \\
\text { desenvolvimento } \\
\text { cognitivo }\end{array}$ & $\begin{array}{l}\text { - Aspectos do desenvolvimento global do aluno com NEE } \\
\text { (cognitivo; social; psicomotor; afetivo) } \\
\text { - A importância da família no processo de ensino e } \\
\text { aprendizagem } \\
\text { - Etapas da aprendizagem }\end{array}$ & Psicopedagoga \\
\hline $\begin{array}{l}\text { - Desenvolvimento da } \\
\text { linguagem oral }\end{array}$ & $\begin{array}{l}\text { - Definições: língua, linguagem e fala } \\
\text { - Desenvolvimento normal da linguagem oral } \\
\text { - Implicações do desenvolvimento da linguagem oral } \\
\text { no deficiente físico e auditivo }\end{array}$ & Fonoaudióloga \\
\hline $\begin{array}{l}\text { - Etiologia das } \\
\text { deficiências }\end{array}$ & $\begin{array}{l}\text { - Definição de deficiência } \\
\text { - Caracterização da deficiência física e auditiva } \\
\text { - Etiologia das deficiências física e auditiva: causas } \\
\text { genéticas e ambientais }\end{array}$ & Fonoaudióloga \\
\hline $\begin{array}{l}\text { - Aprendizagem da } \\
\text { leitura e da escrita de } \\
\text { alunos com NEEs }\end{array}$ & $\begin{array}{l}\text { - Refletir sobre a concepção tradicional do ensino da } \\
\text { leitura e da escrita } \\
\text { - Conhecer as novas tendências educacionais no } \\
\text { ensino da escrita para alunos com NEEs; } \\
\text { - Analisar e identificar os níveis de desenvolvimento } \\
\text { do aluno NEE na aquisição da escrita de acordo com a } \\
\text { Proposta de Emília Ferreiro }\end{array}$ & Pedagoga \\
\hline $\begin{array}{l}\text { - Vivências } \\
\text { psicoterápicas com } \\
\text { familiares }\end{array}$ & $\begin{array}{l}\text { - Possibilitar um espaço para discutir as angústias dos } \\
\text { familiares de deficientes } \\
\text { - Trabalho compartilhado } \\
\text { - Técnicas de relaxamento } \\
\text { - Autoconhecimento das questões familiares } \\
\text { - Promover a integração entre familiares }\end{array}$ & Psicóloga \\
\hline
\end{tabular}

Portanto, decidimos entrevistar os professores a respeito da vida escolar do aluno deficiente.

Ressalta-se que, dos alunos que recebiam os atendimentos pedagógicos no Cees, nas áreas de deficiência física e auditiva, 3 não estavam matriculados em nenhuma modalidade educacional. Assim, foram realizadas entrevistas com os professores de 27 alunos, perfazendo um total de 12 escolas visitadas, na medida em que vários alunos estudavam na mesma unidade escolar.

As entrevistas foram realizadas durante a hora de trabalho pedagógico coletivo (HTPC), nas escolas em que os professores lecionavam. Seu roteiro contemplava informações sobre: problemas de comportamento, dificuldades de aprendizagem, adaptação de recursos pedagógicos e participação dos pais no cotidiano acadêmico. Além das entrevistas, foi preciso consultar alguns documentos administrativos, objetivando levantar: a data de ingresso do aluno na escola, modalidade de ensino freqüentada, freqüência às aulas, período e série de matrícula. 
$\mathrm{Na}$ análise dos dados coletados nas entrevistas, percebemos que $15 \%$ dos alunos deficientes apresentam problemas de comportamento. No entanto, essa situação parece inverter-se quando se trata de investigação sobre os problemas e/ou dificuldades de aprendizagem, pois, segundo os professores entrevistados, $56 \%$ dos alunos apresentavam dificuldades de aprendizagem. Isso pode ser notado no que concerne às dificuldades de realização de atividades de leitura, escrita e noções matemáticas.

Embora a análise dos dados tenha revelado um alto índice de problemas de aprendizagem, por outro lado também demonstrou não ocorrer nenhum tipo de adaptação para sanar essas problemáticas, uma vez que a maioria das respostas dos professores apontou para a ausência de adaptação de recursos, na classe.

Em relação à participação das famílias nas atividades do espaço escolar, percebemos que os professores avaliaram de maneira positiva, pois $71 \%$ da amostra investigada se fez presente quando solicitada.

No que diz respeito à escolaridade dos alunos, podemos dizer que $70 \%$ freqüentam sala especial e/ou sala de recursos e $30 \%$ estão matriculados no ensino regular. Destacamos, ainda, que $56 \%$ encontram-se matriculados nas primeiras séries do Ensino Fundamental, $11 \%$ apenas estão cursando da 5. ${ }^{a}$ a 8. ${ }^{a}$ séries e $33 \%$ estão freqüentando o ensino pré-escolar.

A partir desses resultados, pudemos estabelecer um panorama da situação escolar dos alunos que freqüentam o Cees e esclarecer as dúvidas dos familiares quanto ao atendimento educacional de seus filhos.

\section{Considerações finais}

Após a implementação do programa de orientações psicoeducacionais a familiares e, conseqüentemente, após o acompanhamento das atividades realizadas, podemos destacar que:

- a orientação aos familiares não se restringiu à área educacional, uma vez que o desenvolvimento das temáticas escolhidas possibilitou também a compreensão, por parte dos familiares, de questões psicossociais referentes ao desenvolvimento global de indivíduos com necessidades educacionais especiais;

- a população investigada confirmou a carência de programas de orientações psicoeducacionais em serviços públicos, manifestando 
dificuldade em ter acesso a profissionais e locais que oferecem esse tipo de suporte;

- por mais que as políticas educacionais atuais apontem para a inclusão educacional do aluno com necessidades educacionais especiais, nesse estudo evidenciou-se uma contradição, pois a maioria dos alunos investigados, com deficiência física e auditiva, encontra-se matriculada em classes especiais, não estando inserida no ensino regular, conforme prevê a LDB 9.394/96 e a Resolução CNE/ CEB $2 / 2001$.

Finalizando, podemos relatar que os resultados obtidos nesse trabalho vêm reafirmar a importância de se implementar propostas de orientações com familiares, aliadas aos atendimentos com os indivíduos que apresentam necessidades educacionais especiais, como na literatura pesquisada. De acordo como proposto na abordagem sistêmica, entende-se que a intervenção psicoeducacional só será efetivada com sucesso quando deixar de focar apenas o indivíduo e se estender aos seus pares, principalmente àqueles que convivem diretamente com ele (MARCHESI; MARTIN, 1996).

\section{REFERÊNCIAS}

BASSEDAS, E. Intervenção educativa e diagnóstico psicopedagógico. Porto Alegre: Artes Médicas, 1996.

BRASIL. Lei de Diretrizes e Bases da Educação Nacional: nova LDB (Lei n. 9.394/ 96). Rio de Janeiro: Qualitymark, 1997.

BRASIL. Diretrizes nacionais para a educação especial na educação básica. Brasília: Ministério da Educação, Secretaria de Educação Especial - MEC/SEESP, 2001.

CONSELHO NACIONAL DE EDUCAÇÃO. CÂMARA DE EDUCAÇÃO BÁSICA. Resolução CNE/CBE n. 2 de 2001. Diário Oficial da União, Brasília, 14 set. 2001. Sec. 1E, p. 39-40.

CONSELHO NACIONAL DA SAÚDE. Decreto n. 939.333, de 14 de janeiro de 1987. Resolução n. 196/96 sobre a pesquisa envolvendo seres humanos. Bioética, v. 7, n. 2, 1996. Suplemento.

CORREA, L. M.; SERRANO, A. M. Envolvimento parental do aluno com necessidades educativas especiais. In: CORREA, L. M. Alunos com necessidades educativas especiais nas classes regulares. Portugal: Porto Editora, 1999. p. 145-157. 
FONSECA, V. da. Envolvimento dos pais e educação primária. In: Educação especial: programa de estimulação precoce. Porto Alegre: Artemed, 1995.

MARCHESI, A.; MARTIN, E. Da terminolgia do distúrbio às necessidades educacionais especiais. In: COLL, C.; PALACIOS, J.; MARCHESI, A. Desenvolvimento psicológico e educação. Porto Alegre: Artes Médicas, 1996.

SOMMERSTEIN, L. C.; WESSELS, M. R. Conquistando e utilizando o apoio da família e da comunidade para o ensino inclusivo. In: STAINBACK, S.; STAINBACK, W. Um guia para educadores. Porto Alegre: Artes Médicas, 1999. p. 414-431.

STAINBACK, S.; STAINBACK, W. Colaboração, rede de apoio e construção de comunidade. In: . Um guia para educadores. Porto Alegre: Artes Médicas, 1999. p. 223-232.

VYGOTSKY, L. S. Psicologia pedagógica. São Paulo: Martins Fontes, 2001.

Texto recebido em 13 maio 2004 Texto aprovado em 14 de set. 2004 